\title{
Modification of HLA Expression as a Possible Factor in the Pathogenesis of Burkitt's Lymphoma
}

\author{
R. Voltz, W. Jilg, and H. Wolf
}

\section{Introduction}

According to the Kiel classification, Burkitt's lymphoma (BL) is a high-grade malignant non-Hodgkin's lymphoma of the B-cell lineage. In all the BL cells one finds a gene translocation which can activate the c-myc oncogen. In addition, $95 \%$ of the endemic-type BLs are infected with the Epstein-Barr virus (EBV). In vitro infection with EBV can transform peripheral blood B-cells into immortalized nonmalignant lymphoblastoid cell lines (LCLs). LCLs can also grow out spontaneously from peripheral blood lymphocytes of EBV-positive donors after the removal of $T$ cells or their inhibition by cyclosporin $\mathrm{A}$ in vitro. In vivo EBV-infected cells are normally controlled by the immune system. Immunosuppression leads to a higher risk of EBV-associated oligoclonal lymphoma. In vitro cytotoxic chromium release assays performed with pairs of BL cells and LCLs of the same BL patient have shown that BLs are not recognized by cytotoxic $T$ cells (CTLs) in contrast to LCLs [1]. Most of the EBV-specific CTLs are HLA class I restricted; there are, however, up to $30 \%$ of class II restricted CTLs in the EBV system. One possibility for the BL cells not being recognized is the altered surface expression of EBV-specific structures which have been functionally termed lymphocyte-detected membrane antigen (LYDMA). One of the possible candidates for LYDMA is the latent membrane protein LMP of the reading

Max von Pettenkofer Institute, University of Munich, Pettenkoferstr. 9a, D-8000 München 2, FRG frame BNLF1, which has been shown to be differentially expressed on BL cells and LCLs [2]. The specific recognition may also be influenced by an altered HLA class I or class II expression as suggested by Torsteindottir et al. [3]. We therefore studied the HLA expression on eight pairs of BL cells and LCLs.

\section{Results}

For measuring the HLA density we used a radioimmunoassay with monoclonal antibodies against a framework determinant of class I (clone W6/32) or class II (clone L243) antigen and ${ }^{125}$ I-labeled protein A. To correct for differences in surface area, cells were also incubated with saturating amounts of a mixture of polyclonal antisera against BL cells and LCLs; the cpm values obtained with the monoclonal antibodies were divided by the cpm values of the polyclonal serum mixture, leading to values of relative density of class I and II antigen. Most of the pairs tested showed a significantly lower HLA class I (Fig. 1) and class II density (data not shown) on the BL cells. EBVpositive LCLs showed a much higher HLA density than peripheral blood Bcells of the same donor (data not shown). In contrast to the corresponding LCLs, both in EBV-positive and -negative BL cells the HLA expression could be stimulated by a factor $3-5$ by IFN- $\gamma$ (Fig. 2) and TNF (data not shown). TNF did not show a direct toxic effect against BL cells or LCLs (data not shown).

After immunoprecipitation we performed a biochemical separation of HLA class I and II antigens by one-dimension- 


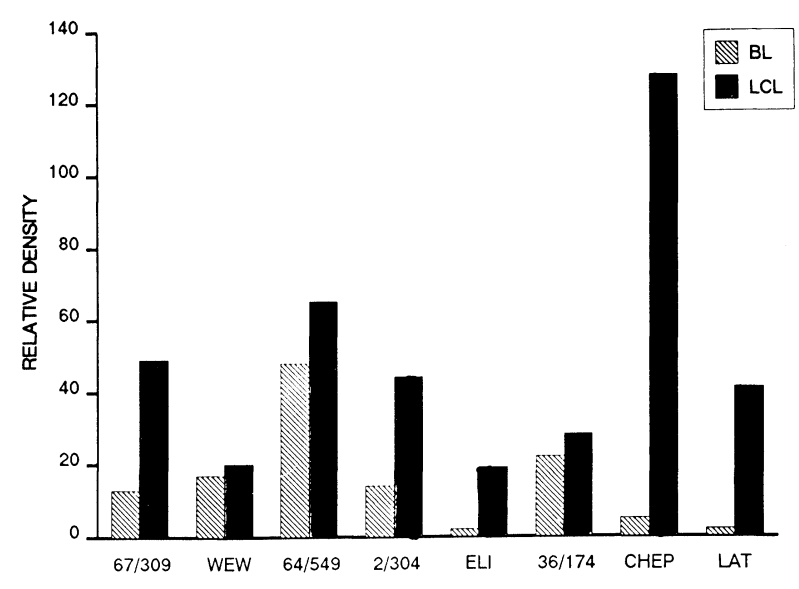

Fig. 1. Expression of class I antigens in BL cells and corresponding LCLs

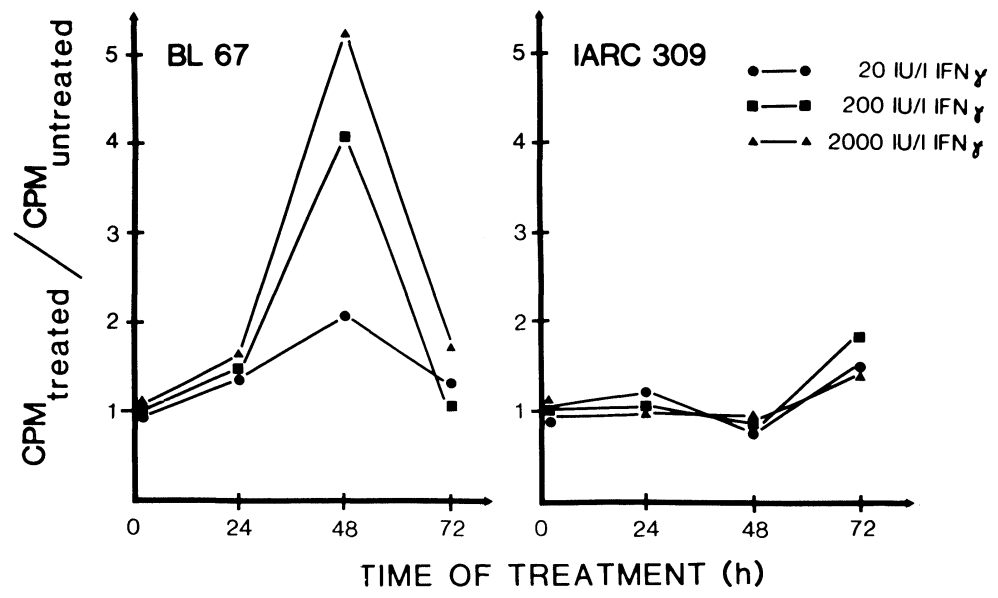

Fig. 2. HLA class I expression under stimulation with IFN- $\gamma$

al SDS-PAGE and two-dimensional electrophoresis which separates according to the isoelectric point and molecular weight. BL cells and LCLs of the same patient showed a different pattern: some of the HLA specificities expressed on LCLs were not found - or some only in traces - on the corresponding BL cells (Fig. 3). After incubation with tunicamycin preventing glycosylation of proteins, these differences were still detected, indicating that they are not due to differences in glycosylation in BL cells and LCLs (data not shown). These differences could not be affected by IFN- $\gamma$ or TNF (data not shown). EBV infection itself did not alter HLA expression qualitatively. This was shown by a comparison of EBV-negative pokeweed mitogenstimulated B-blasts and corresponding EBV-positive LCLs and a comparison of an EBV-negative BL-cell line before and after infection with EBV (data not shown). 
$B L$

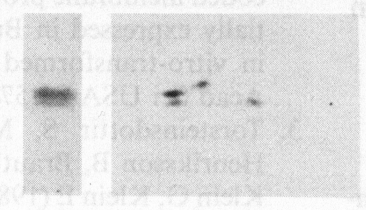

$02 / 304$
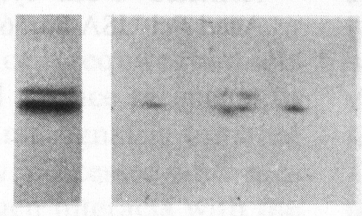

$36 / 174$
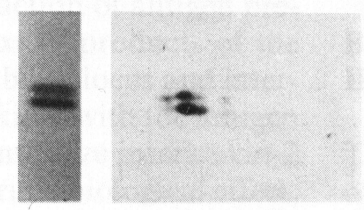

$64 / 549$
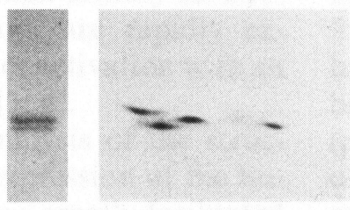

$67 / 309$

Fig. 3. SDS PAGE and two-dimensional gel electrophoresis of HLA class I immunoprecipitates of five BL/ LCL pairs
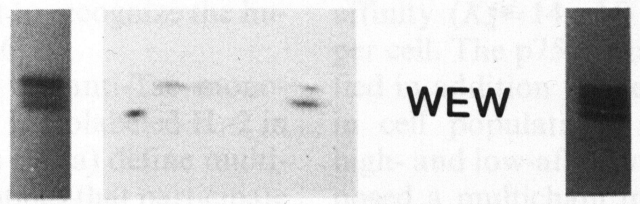
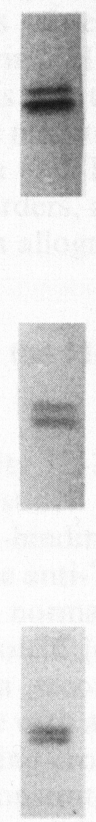

LCL
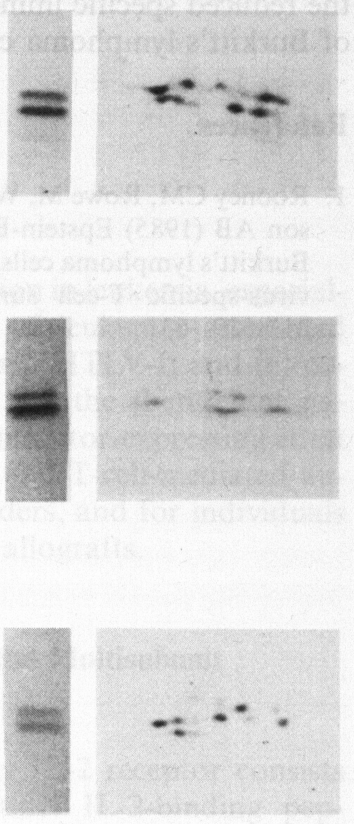

WEW

\section{Summary and Conclusions}

Our data show that

- Most BL cells express significantly less HLA class I and II than the corresponding LCLs.

- Lymphoblastoid cell lines have a much higher HLA class I and II density than normal peripheral blood B cells.
- There are qualitative differences in class I and II expression in most BL/ LCL pairs.

- These qualitative differences are not caused by EBV infection. They might be due to a selective downregulation of HLA specifities by c-myc activation. 
We conclude that these modifications of HLA expression may well play a role in the reduced specific immune recognition of Burkitt's lymphoma cells.

\section{References}

1. Rooney CM, Rowe M, Wallace LE, Rickinson AB (1985) Epstein-Barr virus-positive Burkitt's lymphoma cells not recognized by virus-specific T-cell surveillance. Nature 317:629-631
2. Modrow S, Wolf H (1986) Characterization of two related Epstein-Barr virus-encoded membrane proteins that are differentially expressed in Burkitt lymphoma and in vitro-transformed cell lines. Proc Natl Acad Sci USA 85:5703-5707

3. Torsteinsdottir S, Masucci MG, EhlinHenriksson B, Brautbar C, Ben Bassat H, Klein G, Klein E (1986) Differentiation-dependent sensitivity of human B-cell-derived lines to major histocompatibility complexrestricted T-cell cytotoxicity. Proc Natl Acad Sci USA 83:5620-5624 